\section{An Extension of LaSalle's Invariance Principle and Its Application to Multi-Agent Consensus}

Daizhan Cheng, Jinhuan Wang, and Xiaoming $\mathrm{Hu}$

\begin{abstract}
In the paper, an extension of LaSalle's Invariance Principle to a class of switched linear systems is studied. One of the motivations is the consensus problem in multi-agent systems. Unlike most existing results in which each switching mode in the system needs to be asymptotically stable, this paper allows that the switching modes are only Lyapunov stable. Under certain ergodicity assumptions, an extension of LaSalle's Invariance Principle for global asymptotic stability is obtained. Then it is used to solve the consensus reaching problem of certain multi-agent systems in which each agent is modeled by a double integrator, and the associated interaction graph is switching and is assumed to be only jointly connected.
\end{abstract}

Index Terms-LaSalle's invariance principle, multi-agent consensus, switched linear systems, weak common quadratic Lyapunov function.

\section{INTRODUCTION}

Consider a switched linear system

$$
\dot{x}=A_{\sigma(t)} x, x \in \mathbb{R}^{n}
$$

where $\sigma:[0,+\infty) \rightarrow \Lambda=\{1,2, \ldots, N\}$ is a right continuous piecewise constant function, called a switching signal (or switching law). We use $\varphi\left(x_{0}, t\right)$ for the solution of (1) with a fixed switching law and initial condition $\varphi(0)=x_{0}$. For determining the stability of system (1), it is very natural for us to search for a common quadratic Lyapunov function (CQLF), $V(x)=x^{T} P x$, where $P>0$ is a positive definite matrix, such that

$$
P A_{\lambda}+A_{\lambda}^{T} P<0, \quad \forall \lambda \in \Lambda \text {. }
$$

Finding a CQLF is an interesting and challenging problem. There is a large amount of literature concerning it, e.g., [3], [6], [10] and the references therein. It is worth mentioning that it was shown in [7] that the existence of a common quadratic Lyapunov function is only a sufficient condition for switched linear systems to be asymptotically stable.

The method of multiple Lyapunov functions is also a useful tool for stability analysis of switched systems. In comparison to a common Lyapunov function, it allows each switching mode to have its own Lyapunov function [2].

When the total derivative of the candidate Lyapunov function with respect to each mode is only non-positive, the function is called a weak Lyapunov function [1]. In order to solve the stability problem in such a case, several extensions of LaSalle's invariance principle for hybrid systems have been investigated. For instance, [4] developed two principles, which characterize asymptotic convergence of bounded hybrid trajectories to weakly invariant sets. In [5], an invariance principle was developed for left-continuous dynamic systems. As a special case of

Manuscript received December 20, 2006; revised February 23, 2008. Current version published September 10,2008. This work was supported in part by the National Natural Science Foundation (NNSF) of China under Grants 60221301, 60674022, and 60736022, and by Sida-VR Swedish Research Links Grant 3482006-5894. Recommended by Associate Editor J. Lygeros.

D. Cheng and J. Wang are with the Institute of Systems Science, Chinese Academy of Sciences, Beijing 100080, China (e-mail: dcheng@iss.ac.cn; wangjinhuan@amss.ac.cn).

$\mathrm{X}$. Hu is with the Optimization and Systems Theory and ACCESS Linnaeus Center, Royal Institute of Technology, Stockholm 10044, Sweden, (e-mail: hu@kth.se).

Digital Object Identifier 10.1109/TAC.2008.928332 this result, new invariant set stability theorems were established for nonlinear impulsive dynamic systems. In [8], distinct regularity assumptions on the class of switching signals are placed. Depending on the structural assumptions, either asymptotic stability or simple convergence to an invariant set is concluded. Then the results are extended to nonlinear systems [9]. A more traditional style extension of LaSalle's invariance principle, which emphasizes on set attraction, is proposed in [1]. Under the assumption that the switching signals have a positive average dwell-time, another extension of LaSalle's invariance principle for switched nonlinear systems and criteria for asymptotic stability are obtained in [11].

It has been pointed out that [1], [8] certain restriction on the switching signals is necessary for extending LaSalle's invariance principle. A switched system is said to have a non-vanishing dwell time, if there exists a positive time period $\tau_{0}>0$, such that the switching instances $\left\{\tau_{k} \mid k=1,2, \ldots\right\}$ satisfy

$$
\inf _{k}\left(\tau_{k+1}-\tau_{k}\right) \geq \tau_{0} .
$$

Throughout this paper we assume that

A1 Admissible switching signals have a dwell time $\tau_{0}>0$.

The following is a multi-Lyapunov function approach to LaSalle's invariance principle for switched linear systems.

Proposition 1: [8] Consider system (1). Suppose that there exists a set $\left\{P_{\lambda} \mid \lambda \in \Lambda\right\}$ of $n \times n$ symmetric positive definite matrices and a set of $n \times m$ matrices $\left\{C_{\lambda} \mid \lambda \in \Lambda\right\}$ such that at each switching moment we have

$$
x^{T}(t) P_{\sigma(t)} x(t) \leq x^{T}(t) P_{\sigma\left(t^{-}\right)} x(t), \quad \forall t>0
$$

and

$$
P_{\lambda} A_{\lambda}+A_{\lambda}^{T} P_{\lambda} \leq-C_{\lambda}^{T} C_{\lambda}, \quad \forall \lambda \in \Lambda .
$$

Then (1) is Lyapunov stable. Moreover, if each pair $\left(C_{\lambda}, A_{\lambda}\right)$ is observable, then (1) is asymptotically stable.

Note that if $\left(C_{\lambda}, A_{\lambda}\right)$ is observable and there exists $P_{\lambda}>0$ such that (5) holds, then $A_{\lambda}$ is a Hurwitz matrix ([12]). In this paper, we explore a new extension, which allows Lyapunov stable modes. Of course, in this case some restriction on the switching signal is necessary and we will pose an ergodicity assumption on the switching signals.

In recent years, the study of consensus of multiple agents has attracted much attention, see for example [14]-[18] and the references therein. For many typical models of multi-agent systems adopted in the literature, it appears that when local information is used the closed-loop switching modes can only be Lyapunov stable. This makes it impossible to use the existing extensions of LaSalle's invariance principle to solve the consensus problem for such a system, and partially motivates our study. Later on, one sees that the ergodicity assumption we will pose is satisfied if the interaction graphs of the multi-agent system are jointly connected.

The rest of the paper is organized as follows: Section II introduces a new kind of weak Lyapunov functions, the so-called common joint quadratic Lyapunov function (CJQLF). In Section III an extension of LaSalle's invariance principle is proposed. Section IV is the application of the result obtained in Section III to the consensus problem of multiagent systems. Section V is the conclusion.

\section{JOINT QUADRATIC LYAPUNOV FUNCTION}

In this section, we propose a new concept, called the joint quadratic Lyapunov function. By using it we will develop an extension of LaSalle's invariance principle for system (1). 
Since we only require each mode to be Lyapunov stable, in addition to $\mathbf{A 1}$, we need to assume certain ergodicity property for the switching signal.

A2. There exists a $T>0$, such that for any $t_{0} \geq 0$, the switching signal $\sigma(t)$ satisfies

$$
\{t \mid \sigma(t)=\lambda\} \bigcap\left[t_{0}, t_{0}+T\right] \neq \emptyset, \quad \forall \lambda \in \Lambda .
$$

Remark: If both $\mathrm{A} 1$ and $A 2$ hold, then there exists $T>0$ (replacing the original $T$ of $A 2$ by $\left.T+\tau_{0}\right)$ such that

$$
\left|\{t \mid \sigma(t)=\lambda\} \bigcap\left[t_{0}, t_{0}+T\right]\right| \geq \tau_{0}, \quad \forall \lambda \in \Lambda, \quad t_{0} \geq 0
$$

where $|\cdot|$ denotes the Lebesgue measure.

Definition 1: Consider system (1).

1) If a quadratic function $V(x)=x^{T} P x$ with positive definite $P>$ 0 has the following property:

$$
P A_{i}+A_{i}^{T} P=-Q_{i} \leq 0, \quad i=1, \ldots, N
$$

then $V(x)$ (or briefly, $P$ ) is called a common weak quadratic Lyapunov function (CWQLF) of system (1).

2) A common weak quadratic Lyapunov function of system (1) is called a common joint quadratic Lyapunov function (CJQLF) if

$$
Q:=\sum_{i=1}^{N} Q_{i}>0
$$

Remark: It is easy to show that for system (1), if there exists a CWQLF $P>0$, then $P$ is a CJQLF if and only if

$$
\bigcap_{i \in \Lambda} Z_{i}=\{0\}
$$

where $Z_{i}$ is the kernel of $Q_{i}, i \in \Lambda$.

Now, a fundamental question is: under the assumptions of $\mathrm{A} 1$ and $\mathrm{A} 2$, is the existence of a CJQLF sufficient for assuring the global asymptotical stability?

Unfortunately, the answer is negative and the following is a counter example:

1) Example 1: Consider a switched linear system

$$
\dot{x}=A_{\sigma(t)} x
$$

with a right continuous piecewise constant switching law $\sigma(t)$ : $[0,+\infty) \rightarrow \Lambda=\{1,2,3\}$. Let

$$
\begin{aligned}
A_{1} & =\left(\begin{array}{ccc}
0 & 1 & 0 \\
-1 & 0 & 0 \\
0 & 0 & -1
\end{array}\right), A_{2}=\left(\begin{array}{ccc}
0 & 0 & -1 \\
0 & -1 & 0 \\
1 & 0 & 0
\end{array}\right) \\
A_{3} & =\left(\begin{array}{ccc}
-1 & 0 & 0 \\
0 & 0 & 1 \\
0 & -1 & 0
\end{array}\right) .
\end{aligned}
$$

Choose $P=I_{3}$. A straightforward computation shows that

$$
\begin{aligned}
& Q_{1}=\operatorname{diag}\{0,0,2\}, \quad Q_{2}=\operatorname{diag}\{0,2,0\} \\
& Q_{3}=\operatorname{diag}\{2,0,0\},
\end{aligned}
$$

and $Z_{1}=\operatorname{span}\left\{(1,0,0)^{T},(0,1,0)^{T}\right\}, \quad Z_{2}=$ $\operatorname{span}\left\{(1,0,0)^{T},(0,0,1)^{T}\right\}, Z_{3}=\operatorname{span}\left\{(0,1,0)^{T},(0,0,1)^{T}\right\}$.
Obviously, $Q_{i} \geq 0, i=1,2,3$, and $Q=\sum_{i=1}^{3} Q_{i}=2 I_{3}>0$, namely $I_{3}$ is a common joint quadratic Lyapunov function. We choose a periodic switching law with period $T=1.5 \pi$

$$
\sigma(t)= \begin{cases}1, & t \in[k T, k T+0.5 \pi) \\ 2, & t \in[k T+0.5 \pi, k T+\pi) \\ 3, & t \in[k T+\pi,(k+1) T), k=0,1, \ldots\end{cases}
$$

which is ergodic. However, system (11) is not asymptotically stable under the above switching law if we take the initial value $x(0)=$ $(0,1,0)^{T} \in Z_{1}$. Indeed, the trajectory is a closed loop with $\|x(t)\| \equiv 1$ for all $t \geq 0$.

Therefore, in addition to A1, A2, and the existence of CJQLF, some additional conditions may be needed to assure that the system is globally asymptotically stable, which will be discussed in Section III.

Before ending this section, we give a brief discussion on attractive sets.

Definition 2: [1] A compact set $M$ is weakly invariant with respect to (1), if for each point $x \in M$, there exists a $\lambda \in \Lambda$ and $b>0$ such that the trajectory of $\exp \left(A_{\lambda} t\right) x \in M$ for either $t \in[0, b]$ or $t \in[-b, 0]$. With some mild modification, we state Theorem 1 of [1] as

Proposition 2: [1] Assume system (1) has a CWQLF

$$
z=\bigcup_{i \in \lambda} z_{i}
$$

and $M$ is the largest weak invariant set contained in $Z$. Then every solution $\varphi\left(x_{0}, t\right)$ of system (1) converges to $M$.

\section{LASALLE'S INVARIANCE PRINCIPLE FOR A ClaSS OF $A_{i}$}

In this section, we impose certain constraints on system (1), or more precisely, on $\left\{A_{i}\right\}$. We begin with the following observation.

Lemma 1: For system (1) if there exists a CWQLF, then for each $i$, $A_{i}$ is Lyapunov stable, which implies that $A_{i}$ can only have eigenvalues with non-positive real part. Moreover, the algebraic multiplicity of each eigenvalue on the imaginary axis is equal to its geometric multiplicity.

Definition 3: Assume $P$ is a CWQLF for system (1). Set $z=$ $P^{1 / 2} x$. Then $z$ is called the normal coordinate frame.

Remark: A straightforward computation shows that under the normal coordinate frame $P, A_{i}$ and $Q_{i}$, denoted by $\tilde{P}, \tilde{A}_{i}$ and $\tilde{Q}_{i}$ respectively, become

$$
\begin{aligned}
\tilde{P} & =I_{n}, \quad \tilde{A}_{i}=P^{1 / 2} A_{i} P^{-1 / 2} \\
\tilde{Q}_{i} & =P^{-1 / 2} Q_{i} P^{-1 / 2}, \quad i \in \Lambda .
\end{aligned}
$$

It is worthwhile noting that since $P$ is identity now, the Lyapunov function becomes $\|z\|^{2}$.

In the following discussion we use only the normal coordinate frame unless otherwise stated. To avoid notational mess, instead of $z$, we still use $x$. We need some preparations first.

Lemma 2: Consider a linear system

$$
\dot{x}=A x, \quad x \in \mathbb{R}^{n}
$$

where $A$ is Lyapunov stable. Denote $K=\operatorname{ker}(A)$, and let $y \in K$. Then for any $R>0$, there exists $r>0$, such that if $\left\|x_{0}-y\right\|<r$ then

$$
\left.\| \varphi\left(x_{0}, t\right)-y\right) \|<R, \quad t \geq 0 .
$$

Proof: It is easy to see that any $y \in K$ is a stable equilibrium of (13), thus the conclusion follows. In particular we can choose $r=$ $R / L$, where $L=\max _{t \geq 0}\left\|e^{A t}\right\|<+\infty$. Then

$$
\begin{aligned}
\|x(t)-y\| & =\left\|e^{A t}\left(x_{0}-y\right)\right\| \leq L\left\|x_{0}-y\right\|<L r=R \\
t & \geq 0 .
\end{aligned}
$$

Lemma 3: Consider the switched linear system (1). Assume $A_{i}, i \in$ $\Lambda$ are Lyapunov stable matrices. Denote $K_{i}=\operatorname{ker}\left(A_{i}\right), K=\bigcap_{i \in \Lambda} K_{i}$, 
and let $y \in K$. Assume the switching signal satisfies $A 1$ and $A 2$. Then for any $R>0$, there exists $r>0$, such that if $\left\|x_{0}-y\right\|<r$ then

$$
\left\|\varphi\left(x_{0}, t\right)-y\right\|<R, \quad 0 \leq t \leq T \text {. }
$$

Proof: Let $L=\sup _{t \geq 0, i \in \Lambda}\left\|e^{A_{i} t}\right\|$. Then $L<\infty$ since every $A_{i}$ is Lyapunov stable. Let $k=\left[T / \tau_{0}\right]+1$. Then there are at most $k$ switches over the duration $0 \leq t \leq T$. Choose $r=R / L^{k+1}$.

Let $\lambda_{0}, \lambda_{1}, \ldots \lambda_{s}$ be the sequential active modes with lasting times $t_{0}, t_{1}, \ldots t_{s}$ respectively. Then $s \leq k$ and

$$
\begin{aligned}
\left\|\varphi\left(x_{0}, t\right)-y\right\| & =\left\|e^{A_{\lambda_{s}} t_{s}} \cdots e^{A_{\lambda_{0}} t_{0}} x_{0}-y\right\| \\
& =\left\|e^{A_{\lambda_{s}} t_{s}} \cdots e^{A_{\lambda_{0}} t_{0}}\left(x_{0}-y\right)\right\| \\
& \leq L^{k+1} r=R .
\end{aligned}
$$

Remark: From the proof of Lemma 3, one sees easily that the Lemma remains true if $\Lambda$ is replaced by $\Lambda^{\prime}=\{\lambda \mid$ Mode $\lambda$ is active for some $t \in[0, T]$. $\}$.

Lemma 4: $\operatorname{ker}\left(A_{i}\right) \subseteq \operatorname{ker}\left(Q_{i}\right)$.

Proof: Since

$$
A_{i}+A_{i}^{T}=-Q_{i}
$$

then, for any $x_{0} \in \operatorname{ker}\left(A_{i}\right)$,

$$
x_{0}^{T} Q_{i} x_{0}=-x_{0}^{T}\left(A_{i}+A_{i}^{T}\right) x_{0}=0 .
$$

So $Q_{i} x_{0}=0$ due to the fact that $Q_{i} \geq 0$. The conclusion follows.

Denote by $M$ the largest weak invariant set contained in $Z$, and let

$$
V_{i}=M \bigcap Z_{i}, \quad i=1, \ldots, N \text {. }
$$

Using Lemma 4, one sees easily that $\operatorname{ker}\left(A_{i}\right)$ itself is a weak invariant set contained in $Z_{i} \subset Z$, hence $\operatorname{ker}\left(A_{i}\right) \subseteq V_{i}$. We further assume

A3. $\operatorname{ker}\left(A_{i}\right)=V_{i}, i \in \Lambda$.

Now we are ready to present our main result.

Theorem 1: Consider system (1). Assume $A 1$ and $A 2$ hold, and there exists a CJQLF such that $A 3$ holds. Then system (1) is globally asymptotically stable.

Proof: Choosing $x_{0} \in \mathbb{R}^{n}$ and let $x(t)=\varphi\left(x_{0}, t\right)$ be any trajectory of system (1) under the normal coordinate frame and with certain switch $\sigma$. Since $\left\|\varphi\left(x_{0}, t\right)\right\|$ is non-increasing monotonically, the Lyapunov stability is obvious. Moreover

$$
\lim _{t \rightarrow \infty}\left\|\varphi\left(x_{0}, t\right)\right\|=c
$$

Thus we only need to show $c=0$. We will prove this by contradiction. Assume $c>0$. Since $x(t)$ is bounded, then there exists an infinite sequence $\left\{t_{k}\right\}$ such that

$$
x_{k}:=x\left(t_{k}\right) \rightarrow y \text { as } k \rightarrow \infty
$$

where $\|y\|=c>0$. Now since $y$ is a point in the $\omega$-limit set (see [1]), we have $y \in M \subset Z$.

Split $\Lambda$ into two disjoint subsets, $I \subset \Lambda$ and $J=\Lambda \backslash I$, such that

$$
y \in Z_{i}, \quad \forall i \in I, \quad y \notin Z_{j}, \quad \forall j \in J .
$$

Since $y \in M$, thus $I \neq \emptyset$ and $y \in V_{i}, \forall i \in I$. According to condition (10), $J \neq \emptyset$ either.
Denote

$$
d=\min _{j \in J} d\left(y, Z_{j}\right)>0 .
$$

We can choose $0<R<d / 2$ and define a ball $B_{R}(y)$ (where $B_{r}(p)=$ $\left\{x \in \mathbb{R}^{n} \mid\|x-p\|<r\right\}$ ). Then we have

$$
d\left(x, Z_{j}\right)>R, \quad x \in B_{R}(y), \quad \forall j \in J .
$$

For each $x \in \mathbb{R}^{n}$, we can decompose it with respect to each $i \in \Lambda$ by $x=x_{1}+x_{2}$, where $x_{1} \in Z_{i}$ and $x_{2} \in Z_{i}^{\perp}$. If mode $i$ is active, then

$$
\frac{d}{d t}\|x(t)\|^{2}=,-x_{2}^{T} Q_{i} x_{2}
$$

and

$$
\sigma_{m} x_{2}^{T} x_{2} \leq x_{2}^{T} Q_{i} x_{2} \leq \sigma_{M} x_{2}^{T} x_{2}
$$

where $\sigma_{m}$ and $\sigma_{M}$ are the smallest and largest nonzero (equivalently, positive) eigenvalues of $Q_{i}$, and $\left\|x_{2}\right\|$ is the distance of $x$ to $Z_{i}$.

Let

$\mu_{\min }=\min _{j \in J}\left\{\lambda_{k}^{j} \mid \lambda_{k}^{j}:\right.$ nonzero eigenvalues of $\left.Q_{j}\right\}>0$.

Then it is clear that if a mode $j \in J$ is active, then

$$
\begin{aligned}
\frac{d}{d t}\|x(t)\|^{2} & =-x^{T}(t) Q_{j} x(t) \\
& \leq-\mu_{\min }\left\|x_{2}\right\|^{2} \\
& \leq-\mu_{\min } R^{2}, \quad \forall x \in B_{R}(y) .
\end{aligned}
$$

Choose a positive number $N_{0} \gg 1$ and let $R_{1}^{0}=R / N_{0}$. Then we can find an $\alpha>0$ such that

$$
\max _{j \in J}\left\|e^{A_{j} t}-I\right\| \leq \frac{\left(N_{0}-1\right) R_{1}^{0}}{\|y\|+R_{1}^{0}}, \quad 0 \leq t \leq \alpha .
$$

Since when $\alpha=0$ the left hand side of (21) is zero, (21) follows from the continuity. Without loss of generality we can assume $\alpha \leq \tau_{0}$.

Now assume for the time being that we can find a moment $t_{0}$ such that $x\left(t_{0}\right) \in B_{R_{1}^{0}}(y)$ and at $t_{0}$ a mode $j \in J$ is triggered, then we have

$$
\begin{aligned}
\|x(t)-y\| & \leq\left\|x(t)-x\left(t_{0}\right)\right\|+\left\|x\left(t_{0}\right)-y\right\| \\
& \leq \frac{\left(N_{0}-1\right) R_{1}^{0}}{\|y\|+R_{1}^{0}}\left\|x\left(t_{0}\right)\right\|+R_{1}^{0} \\
& \leq R, \quad t_{0} \leq t \leq t_{0}+\alpha .
\end{aligned}
$$

Denote $\delta=\mu_{\min } R^{2} \alpha>0$. From (22) and the fact that the mode $j \in J$ becomes active at $t_{0}$, we can use (20) to get

$$
\begin{aligned}
\left\|x\left(t_{0}+\alpha\right)\right\|^{2} & <\left\|x\left(t_{0}\right)\right\|^{2}-\delta \\
& <\left\|x\left(t_{0}\right)\right\|^{2}-\delta+\left(\frac{\delta}{2\left\|x\left(t_{0}\right)\right\|}\right)^{2} \\
& =\left(\left\|x\left(t_{0}\right)\right\|-\frac{\delta}{2\left\|x\left(t_{0}\right)\right\|}\right)^{2} .
\end{aligned}
$$

Hence

$$
\left\|x\left(t_{0}+\alpha\right)\right\| \leq\left\|x\left(t_{0}\right)\right\|-\frac{\delta}{2\left\|x\left(t_{0}\right)\right\|} .
$$


Since $x\left(t_{0}\right) \in B_{R_{1}^{0}}(y)$, then $\left\|x\left(t_{0}\right)\right\|<\|y\|+R_{1}^{0}<\|y\|+R$, and hence

$$
\left\|x\left(t_{0}+\alpha\right)\right\|<\left\|x\left(t_{0}\right)\right\|-\frac{\delta}{2(\|y\|+R)} .
$$

Now choose

$$
R_{1}=\min \left(R_{1}^{0}, \quad \frac{\delta}{2(\|y\|+R)}\right)
$$

and let $x\left(t_{0}\right) \in B_{R_{1}}(y) \subseteq B_{R_{1}^{0}}(y)$. Then (24) yields

$$
\left\|x\left(t_{0}+\alpha\right)\right\|<\|y\|+R_{1}-\frac{\delta}{2(\|y\|+R)} \leq\|y\|
$$

i.e., $\left\|x\left(t_{0}+\alpha\right)\right\|<\|y\|$, which is a contradiction because $\|y\|=c$ is the lower bound of $\|x(t)\|$.

Finally, we have to show that for $R_{1}$ given by (25), the required $t_{0}$ in the previous assumption exists. Since $y$ belongs to the $\omega$-limit set, for any $r>0$ there exists $t^{*}$ such that $x\left(t^{*}\right) \in B_{r}(y)$. Using the remark after Lemma 3 together with Assumption $A 3$, we can find $0<r<R_{1}$ such that when $x\left(t^{*}\right) \in B_{r}(y)$ and only some modes $i \in I$ are active, we have

$$
\varphi\left(x_{0}, t\right) \in B_{R_{1}}(y), \quad t^{*} \leq t \leq t^{*}+T .
$$

Recalling Assumption $A 2$, a $j \in J$ mode will become active at some moment $t_{0} \in\left[t^{*}, t^{*}+T\right]$ and by (27) we know that the trajectory $\varphi\left(x_{0}, t\right)\left(t^{*} \leq t \leq t_{0}\right)$ remains in $B_{R_{1}}(y) \subseteq B_{R_{1}^{0}}(y)$.

In general, it is not straightforward to verify A3. We thus give a sufficient condition here.

Proposition 3: If $\operatorname{rank}\left(A_{i}\right)=\operatorname{rank}\left(A_{i}+A_{i}^{T}\right)$ (equivalently in the original coordinate frame: $\left.\operatorname{rank}\left(A_{i}\right)=\operatorname{rank}\left(P A_{i}+A_{i}^{T} P\right)\right), i=$ $1, \ldots, N$, then A3 is satisfied.

Proof: Lemma 4 implies that $\operatorname{ker}\left(A_{i}\right) \subseteq \operatorname{ker}\left(Q_{i}\right)$. Now since $\operatorname{rank}\left(A_{i}\right)=\operatorname{rank}\left(Q_{i}\right)$, we have $\operatorname{dim}\left(\operatorname{ker}\left(A_{i}\right)\right)=\operatorname{dim}\left(\operatorname{ker}\left(Q_{i}\right)\right)$. Therefore, $\operatorname{ker}\left(A_{i}\right)=\operatorname{ker}\left(Q_{i}\right)=Z_{i}$. Then $V_{i} \subseteq \operatorname{ker}\left(A_{i}\right)$. Meanwhile, since $\operatorname{ker}\left(A_{i}\right)$ is $A_{i}$ invariant and contained in $\operatorname{ker}\left(Q_{i}\right)$, so, $\operatorname{ker}\left(A_{i}\right) \subseteq V_{i}$. We conclude that $V_{i}=\operatorname{ker}\left(A_{i}\right)$.

A particular interesting case is the following:

Remark: If $A_{i}$ (equivalently in the original coordinate frame: $\left.P^{1 / 2} A_{i} P^{-1 / 2}\right), i \in \Lambda$ are symmetric, then $A 3$ is satisfied automatically.

\section{Consensus of Leader Following Multi-Agent Systems}

In this section, we study the consensus problem of multi-agent systems with variable interaction topology by applying the results from the previous sections.

In order to apply our results to the multi-agent consensus problem we need to modify (in fact weaken) Assumption $\mathbf{A 2}$ to the following.

A2' There exists a $T>0$, such that for any $t \geq 0$

$$
\sum_{j \in J[t, t+T]} Q_{j}>0
$$

where $J[t, t+T]:=\{\sigma(s) \mid s \in[t, t+T]\}$.

Remark: In the study of consensus problems a common assumption is that there exist a $T_{0}>0$ and a sequence $0<t_{1}<t_{2}<\cdots \rightarrow$ $\infty, t_{i+1}-t_{i} \leq T_{0}, \forall i$ such that the graphs over $\left[t_{i}, t_{i+1}\right]$ are jointly connected. This requires that

$$
\sum_{j \in J\left[t_{i}, t_{i+1}\right]} Q_{j}>0, \quad \forall i
$$

In fact (28) and (29) are equivalent. Equation (28) $\Rightarrow(29)$ is obvious. To prove (29) $\Rightarrow(28)$, set $T=2 T_{0}$, then for any $t \geq 0$, there exists a $k$ such that $\left[t_{k}, t_{k+1}\right] \subset[t, t+T]$.

Proposition 4: Theorem 1 remains true when Assumption $A 2$ is replaced by $A 2^{\prime}$.

Proof: According to (10), $A 2^{\prime}$ assures that on each time duration $[t, t+T], \forall t \geq 0$, certain $j \in J$ mode must be active. So the argument in the proof of Theorem 1 remains true.

In coordination problems for multiple agents, tools from the algebraic graph theory are used frequently (referring to [13], [19] for the details).

We consider a system consisting of $n$ agents and a leader which is denoted by a graph $\overline{\mathcal{G}}$. It contains $n$ agents (related to an undirected graph $\mathcal{G}$ ) and a leader (labeled by 0 ) with directed edges from some agents to the leader. The set of neighbors of vertex $i$ at time $t$ is denoted by $\mathcal{N}_{i}(t)=\{j \in \mathcal{V}:(i, j) \in \mathcal{E}, j \neq i\}$, where $\mathcal{V}=\{1,2, \ldots, n\}$ and $\mathcal{E}=\{(i, j): i, j \in \mathcal{V}\} \subset \mathcal{V} \times \mathcal{V}$ are the sets of vertices and edges of graph $\mathcal{G}$, respectively.

A subgraph $\mathcal{X}$ of $\mathcal{G}$ is an induced subgraph if two vertices of $\mathcal{V}(\mathcal{X})$ are adjacent in $\mathcal{X}$ if and only if they are adjacent in $\mathcal{G}$. An induced subgraph of $\mathcal{G}$ that is maximal, subject to being connected, is called a component of $\mathcal{G}$. "The graph $\overline{\mathcal{G}}$ is connected" means that at least one agent in each component of $\mathcal{G}$ is connected to the leader. The union graph of a collection of graphs $\left\{\overline{\mathcal{G}}_{1}, \overline{\mathcal{G}}_{2}, \ldots, \overline{\mathcal{G}}_{m}\right\}$, with the same vertex set $\overline{\mathcal{V}}$, is defined as a graph, denoted by $\overline{\mathcal{G}}$, with vertex set $\overline{\mathcal{V}}$ and edge set equaling the union of the edge sets of all the graphs in the collection. The collection of graphs $\left\{\overline{\mathcal{G}}_{1}, \overline{\mathcal{G}}_{2}, \ldots, \overline{\mathcal{G}}_{m}\right\}$ is called jointly connected if the union graph $\overline{\mathcal{G}}$ is connected [14], [15].

For the multi-agent system concerned, the neighborhood for each agent is time-varying. Assume all possible interconnection topologies of graph $\overline{\mathcal{G}}$ are $\overline{\mathcal{G}}_{1}, \overline{\mathcal{G}}_{2}, \ldots, \overline{\mathcal{G}}_{N}$. Following [14], we assume the weights between agents are

$$
a_{i j}(t)= \begin{cases}a_{i j}^{*}, & \text { if } j \in \mathcal{N}_{i}(t) \\ 0, & \text { otherwise }\end{cases}
$$

and

$$
b_{i}(t)= \begin{cases}b_{i}^{*}, & \text { if agent } i \text { is connected to the leader at } t \\ 0, & \text { otherwise }\end{cases}
$$

where $a_{i j}^{*}, b_{j}^{*}(i, j=1, \ldots, n)$ are fixed positive constants.

In this paper, we consider the following double integrator system of $n$ agents:

$$
\left\{\begin{array}{l}
\dot{q}_{i}=p_{i} \\
\dot{p}_{i}=u_{i}
\end{array}\right.
$$

where $q_{i}, p_{i}, u_{i} \in \mathbb{R}, i=1,2, \ldots, n$, denote the position (or angle), velocity (or angular velocity) and control input of agent $i$, respectively.

There does not seem to exist an obvious way to adopt the matrix analysis method used in [15] directly to the second order dynamics. Thus we will use instead the LaSalle's invariance principle approach to solve the consensus problem. In this paper we consider the consensus problem for leader-following multi-agent systems.

The dynamics of the leader is expressed as follows:

$$
\dot{q}_{0}=p_{0}
$$

where $p_{0} \in \mathbb{R}$ is the desired constant velocity known to all agents.

Our control aim is that all the agents follow the leader asymptotically and the desired velocity of all the agents converges to $p_{0}$, namely, $q_{i} \rightarrow$ $q_{0}, p_{i} \rightarrow p_{0}$ as $t \rightarrow \infty$. 
We use the following neighbor-based feedback control law:

$$
\begin{aligned}
u_{i}= & -\sum_{j \in \mathcal{N}_{i}(t)} a_{i j}(t)\left(q_{i}-q_{j}\right)-b_{i}(t)\left(q_{i}-q_{0}\right) \\
& -k\left(p_{i}-p_{0}\right) \\
i= & 1,2, \ldots, n
\end{aligned}
$$

where $k>1$ is a control parameter.

Let $x_{i}=q_{i}-q_{0}, v_{i}=p_{i}-p_{0}, i=1,2, \ldots, n$, then (32) can be rewritten as

$$
\begin{aligned}
u_{i} & =-\sum_{j \in \mathcal{N}_{i}(t)} a_{i j}(t)\left(x_{i}-x_{j}\right)-b_{i}(t) x_{i}-k v_{i} \\
i & =1,2, \ldots, n .
\end{aligned}
$$

Let $\sigma(t):[0, \infty) \rightarrow \Lambda=\{1,2, \ldots, N\}$ be a right continuous piecewise constant switching signal. The Laplacian of graph $\mathcal{G}_{\sigma(t)}$ is defined as $L_{\sigma(t)}=\left(l_{i j}(t)\right) \in \mathbb{R}^{n \times n}$, where

$$
l_{i j}(t)= \begin{cases}\sum_{k \in \mathcal{N}_{i}(t)} a_{i k}(t), & j=i \\ -a_{i j}(t), & j \neq i .\end{cases}
$$

Denote $\epsilon=\left(\epsilon_{1}^{T}, \ldots, \epsilon_{n}^{T}\right)^{T} \in \mathbb{R}^{2 n}, \quad \epsilon_{i}=\left(x_{i}, v_{i}\right)^{T} \in \mathbb{R}^{2}, \quad u=$ $\left(u_{1}, \ldots, u_{n}\right)^{T} \in \mathbb{R}^{n}$.

With (30) and (33), the closed-loop system for the followers can be expressed as:

$$
\left\{\begin{array}{l}
\dot{x}=v \\
\dot{v}=-\left(L_{\sigma(t)}+B_{\sigma(t)}\right) x-k v
\end{array}\right.
$$

where $L_{\sigma(t)}$ is the Laplacian of $\mathcal{G}_{\sigma(t)}$ and $B_{\sigma(t)}=$ $\operatorname{diag}\left(b_{1}(t), \ldots, b_{n}(t)\right)$ is the leader adjacency matrix of $\overline{\mathcal{G}}_{\sigma(t)}$. We use $\sigma(t)$ to emphasize that they change values only when the switchings occur. Putting variables together, we have

$$
\dot{\epsilon}=F_{\sigma} \epsilon
$$

where

$$
\begin{aligned}
& F_{\sigma}=I_{n} \otimes\left(\begin{array}{cc}
0 & 1 \\
0 & -k
\end{array}\right)+H_{\sigma} \otimes\left(\begin{array}{cc}
0 & 0 \\
-1 & 0
\end{array}\right) \\
& H_{\sigma}=L_{\sigma}+B_{\sigma} .
\end{aligned}
$$

Lemma 5: [14] If graph $\overline{\mathcal{G}}_{p}(p \in \Lambda)$ is connected, then the symmetric matrix $H_{p}$ associated with $\overline{\mathcal{G}}_{p}$ is positive definite. Moreover, let $H_{i_{1}}, \ldots, H_{i_{m}}$ be the matrices associated with the graphs $\overline{\mathcal{G}}_{i_{1}}, \ldots, \overline{\mathcal{G}}_{i_{m}}$, respectively. If these graphs are jointly connected, then $\sum_{j=1}^{m} H_{i j}$ is positive definite.

Now, we present our main result of this section.

Theorem 2: Consider system (35). Assume the switching signal $\sigma(t)$ satisfies $\mathrm{A} 1$, and there exists a $T>0$ such that for any $t \geq$ 0 , the collection of the interaction graphs across each interval $[t, t+$ $T]$ is jointly connected, then the consensus is reached asymptotically, namely, $\lim _{t \rightarrow \infty} \epsilon(t)=0$.

Proof: Take a positive definite matrix $P=I_{n} \otimes\left(\begin{array}{ll}k & 1 \\ 1 & 1\end{array}\right)$, where $k>1$, then

$$
\begin{aligned}
Q_{p} & :=-\left(F_{p}^{T} P+P F_{p}\right) \\
& =I_{n} \otimes\left(\begin{array}{cc}
0 & 0 \\
0 & 2(k-1)
\end{array}\right)+H_{p} \otimes\left(\begin{array}{ll}
2 & 1 \\
1 & 0
\end{array}\right), \quad p \in \Lambda .
\end{aligned}
$$

Since $H_{p}, p \in \Lambda$ are positive semi-definite, there exist orthogonal matrices $U_{p}$ such that

$$
U_{p}^{T} H_{p} U_{p}=\left(\begin{array}{cc}
\Lambda_{p} & 0 \\
0 & 0
\end{array}\right), \quad p \in \Lambda
$$

where $\Lambda_{p}=\operatorname{diag}\left\{\lambda_{1}\left(H_{p}\right), \ldots, \lambda_{r_{p}}\left(H_{p}\right)\right\}$ with $\lambda_{i}\left(H_{p}\right), i=$ $1, \ldots, r_{p}$, being the nonzero eigenvalues of $H_{p}$, and $r_{p}>0$ is the rank of $H_{p}$. At the same time

$$
\begin{aligned}
\bar{F}_{p} & :=\left(U_{p} \otimes I_{2}\right)^{T} F_{p}\left(U_{p} \otimes I_{2}\right) \\
& =I_{n} \otimes\left(\begin{array}{cc}
0 & 1 \\
0 & -k
\end{array}\right)+\left(\begin{array}{cc}
\Lambda_{p} & 0 \\
0 & 0
\end{array}\right) \otimes\left(\begin{array}{cc}
0 & 0 \\
-1 & 0
\end{array}\right) \\
\bar{Q}_{p} & :=\left(U_{p} \otimes I_{2}\right)^{T} Q_{p}\left(U_{p} \otimes I_{2}\right) \\
& =I_{n} \otimes\left(\begin{array}{cc}
0 & 0 \\
0 & 2(k-1)
\end{array}\right)+\left(\begin{array}{cc}
\Lambda_{p} & 0 \\
0 & 0
\end{array}\right) \otimes\left(\begin{array}{ll}
2 & 1 \\
1 & 0
\end{array}\right) .
\end{aligned}
$$

Choose

$$
k>\frac{1}{4} \max \left\{\lambda_{i}\left(H_{p}\right), \quad p \in \Lambda, \quad i=1,2, \ldots, r_{p}\right\}+1
$$

then

$$
\left(\begin{array}{cc}
2 \lambda_{i}\left(H_{p}\right) & \lambda_{i}\left(H_{p}\right) \\
\lambda_{i}\left(H_{p}\right) & 2(k-1)
\end{array}\right)>0
$$

which implies that $Q_{p} \geq 0, p \in \Lambda$.

Denote $J[t, t+T]=\{\sigma(s) \mid s \in[t, t+T]\},|J[t, t+T]|=m_{t}$. On each interval $[t, t+T]$, since $\left\{\overline{\mathcal{G}}_{p}, p \in J[t, t+T]\right\}$ is jointly connected, by Lemma $5, \sum_{p \in J[t, t+T]} H_{p}>0, \forall t \geq 0$.

Define $\lambda=\max \left\{\right.$ eigenvalues of $\left.\sum_{p \in J[t, t+T]} H_{p}>0, \forall t \geq 0\right\}$, which is well defined since $\{J[t, t+T], \forall t \geq 0\} \subset 2^{\Lambda}$ is a finite set. Take $k>1 / 4 \max \left\{\lambda, \lambda_{i}\left(H_{p}\right) \mid p \in \Lambda, i=1,2, \ldots, r_{p}\right\}+1$, then

$$
\begin{aligned}
\sum_{p \in J[t, t+T]} Q_{p}= & I_{n} \otimes\left(\begin{array}{cc}
0 & 0 \\
0 & 2 m_{t}(k-1)
\end{array}\right) \\
& +\left(\sum_{p \in J[t, t+T]} H_{p}\right) \otimes\left(\begin{array}{ll}
2 & 1 \\
1 & 0
\end{array}\right)>0 .
\end{aligned}
$$

Therefore, Assumption $A 2^{\prime}$ holds and $P$ is a CJQLF. Furthermore, by (36) and (37), we can easily obtain that $\operatorname{rank}\left(\bar{F}_{p}\right)=\operatorname{rank}\left(\bar{Q}_{p}\right)$, $p \in \Lambda$, which implies A3 is satisfied by observing Proposition 3. Thus, by Proposition $4, \lim _{t \rightarrow \infty} \epsilon(t)=0$.

\section{CONCLUSION}

By introducing the common joint quadratic Lyapunov function an extension of LaSalle's invariance principle was obtained in this paper. Unlike the traditional extensions, our results did not require each switching mode being asymptotically stable, while certain ergodicity restrictions were imposed on the switching signals. Based on the stability result, a consensus problem of multi-agent systems with variable interaction topology was studied. With a neighbor-based feedback control rule for each agent, we proved that a leader-following multi-agent system of two dimensional dynamics for each agent can achieve consensus asymptotically under the assumption that the collection of the interaction graphs is jointly connected.

\section{ACKNOWLEDGMENT}

The authors would like to thank an anonymous reviewer for the very detailed comments on the proof of the main result. 


\section{REFERENCES}

[1] A. Bacciotti and L. Mazzi, "An invariance principle for nonlinear switched systems," Syst. Control Lett., vol. 54, pp. 1109-1119, 2005.

[2] M. Branicky, "Multiple Lyapunov functions and other analysis tools for switched and hybrid systems," IEEE Trans. Automat. Control, vol. 43, no. 4, pp. 475-482, Apr. 1998.

[3] D. Cheng, L. Guo, and J. Huang, "On quadratic Lyapunov function," IEEE Trans. Automat. Control, vol. 48, no. 5, pp. 885-890, May 2003.

[4] V. Chellaboina, S. P. Bhat, and W. M. Haddad, "An invariance principle for nonlinear hybrid and impulsive dynamical systems," Nonlin. Anal., vol. 53, pp. 527-550, 2003.

[5] R. G. Sanfelice, R. Goebel, and A. R. Teel, "Results on convergence in hybrid systems via detectability and on invariance principle," in Proc. 24th IEEE ACC, 2005, pp. 551-556.

[6] R. N. Shorten, K. S. Narendra, and O. Mason, "A result on common quadratic Lyapunov functions," IEEE Trans. Automat. Control, vol. 48, no. 1, pp. 618-621, Jan. 2003.

[7] W. P. Dayawansa and C. F. Martin, "A converse Lyapunov theorem for a class of dynamic systems which undergo switching," IEEE Trans. Automat. Control, vol. 44, no. 4, pp. 751-760, Apr. 1999.

[8] J. P. Hespanha, "Uniform stability of switched linear systems: Extensions of LaSalle's invariance principle," IEEE Trans. Automat. Control, vol. 49, no. 4, pp. 470-482, Apr. 2004.

[9] J. P. Hespanha, D. Liberzon, E. Anglei, and E. D. Sontag, "Nonlinear observability notion and stability of switched systems," IEEE Trans. Automat. Control, vol. 50, no. 2, pp. 154-168, Feb. 2005.

[10] D. Liberzon, J. P. Hespanha, and A. S. Morse, "Stability of switched systems: A Lie-algebraic condition," Syst. Control Lett., vol. 37, no. 3, pp. 117-122, 1999.

[11] J. L. Mancilla-Aguilar and R. A. Garcia, "An extension of LaSalle's invariance principle for switched systems," Syst. Control Lett., vol. 55, pp. 376-384, 2006.

[12] W. J. Rugh, Linear Systems Theory. Upper Saddle River, NJ: Prentice-Hall, 1993.

[13] R. Horn and C. Johnson, Matrix Analysis. New York: Cambridge Univ. Press, 1985.

[14] Y. Hong, L. Gao, D. Cheng, and J. Hu, "Coordination of multi-agent systems with varying interconnection topology using common Lyapunov function," IEEE Trans. Automat. Control, vol. 52, no. 5, pp. 943-948, May 2007.

[15] A. Jadbabaie, J. Lin, and A. S. Morse, "Coordination of groups of mobile autonomous agents using nearest neighbor rules," IEEE Trans. Automat. Control, vol. 48, no. 6, pp. 998-1001, Jun. 2003.

[16] L. Moreau, "Stability of multiagent systems with time-dependent communication links," IEEE Trans. Automat. Control, vol. 50, no. 2, pp. 169-182, Feb. 2005.

[17] R. Olfati-Saber and R. Murray, "Consensus problems in networks of agents with switching topology and time-delays," IEEE Trans. Automat. Control, vol. 49, no. 9, pp. 1520-1533, Sep. 2004.

[18] W. Ren and R. W. Beard, "Consensus seeking in multi-agent systems using dynamically changing interaction topologies," IEEE Trans. Automat. Control, vol. 50, no. 5, pp. 655-661, May 2005.

[19] C. Godsil and G. Royle, Algebraic Graph Theory. New York: Springer-Verlag, 2001.

\section{Growth Conditions for the Global Stability of High-Speed Communication Networks With a Single Congested Link}

\author{
C. King, R. N. Shorten, F. R. Wirth, and M. Akar
}

\begin{abstract}
This note considers the design of transmission control protocol (TCP)-like congestion control protocols for deployment in high-speed communication networks. A basic problem in this area is to design congestion control strategies that probe more aggressively than standard TCP, but which coexist with each other and result in globally stable and equitable network behavior. In this note, we take a first step towards this goal, by formulating the TCP dynamics as a discrete linear system with nonlinear feedback gain. Under the assumption of player synchronisation, conditions for global network stability are derived in the form of growth bounds on the local nonlinear probing functions.
\end{abstract}

Index Terms-Asymptotic stability, congestion control, discrete-time system, nonlinear additive-increase multiplicative-decrease (AIMD).

\section{INTRODUCTION}

Transmission control protocol (TCP), in congestion avoidance mode, is based primarily on Chiu and Jain's [2] additive-increase multiplicative-decrease (AIMD) paradigm for decentralized allocation of a shared resource (e.g., bandwidth) among competing users. With some minor modifications, the AIMD algorithm has served the networking community well over the past two decades and it continues to provide the basic building block upon which today's internet communication is built. Recently, in the context of designing high speed communication networks, several authors have suggested basic modifications to the AIMD algorithm; for example, see [3], [4], [6], [10], and [11]. One idea underlying these modifications is to replace the constant growth rate of window size in standard TCP with an increasing rate. The rationale for this change is that such protocols probe more aggressively for available bandwidth as network capacity increases [3], [11]. These algorithms, which we refer to as nonlinear AIMD (NAIMD), result in networks with different dynamic properties than those employing the basic (linear) AIMD; see [3], [8]. A basic question in the design of NAIMD networks is how to choose the probing action so that the resulting network exhibits desirable properties. Remarkably, despite increasing deployment of these algorithms (e.g., a high-speed TCP algorithm is implemented as part of the Linux operating system), little work has been carried out in this area and basic questions concerning the existence and nature of network equilibria have yet to be addressed.

Our objective in this note is to study basic convergence and stability properties of a class of NAIMD congestion control protocols. In this

Manuscript received August 21, 2006; revised June 18, 2007. Current version published September 10, 2008. Recommended by Associate Editor C. Beck. This work was supported by the Science Foundation Ireland under Grant 04-IN3 and the Hamilton Institute, National University of Ireland.

C. King is with the Department of Mathematics, Northeastern University, Boston MA 02115 USA (e-mail: king@neu.edu).

R. N. Shorten is with the Hamilton Institute, NUI Maynooth, Maynooth, Co. Kildare, Ireland (e-mail: robert.shorten@nuim.ie).

F. R. Wirth was with the Hamilton Institute, National University of Ireland, Maynooth, Ireland. He is now with the Institute for Mathematics, University of Würzburg, Germany (e-mail: wirth@mathematik.uni-wuerzburg.de).

M. Akar was with the Hamilton Institute, National University of Ireland, Maynooth, Ireland. He is now with the Department of Electrical and Electronic Engineering, Boğaziçi University, Bebek,İstanbul, Turkey (e-mail: mehmet.akar@boun.edu.tr).

Color versions of one or more of the figures in this paper are available online at http://ieeexplore.ieee.org.

Digital Object Identifier 10.1109/TAC.2008.928302 